\title{
Mobile EEG reveals functionally dissociable dynamic processes supporting real-world ambulatory obstacle avoidance: Evidence for early proactive control
}

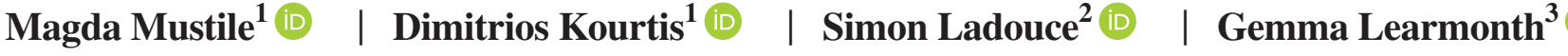 \\ Martin G. Edwards ${ }^{4}$ (i) ｜ David I. Donaldson5 (iD) | Magdalena Ietswaart $^{1}$ (iD
}

${ }^{1}$ Psychology, Faculty of Natural Sciences, University of Stirling, Stirling, UK

${ }^{2}$ Institut Supérieur de l'Aéronautique et de l'Espace (ISAE), Toulouse, France

${ }^{3}$ Institute of Neuroscience \& Psychology, University of Glasgow, Glasgow, UK

${ }^{4}$ Institute of Research in the Psychological Sciences, Université catholique de Louvain, Louvain-la-Neuve, Belgium

${ }^{5}$ School of Psychology and Neuroscience, University of St Andrews, St. Andrews, UK

\section{Correspondence}

Magda Mustile, Psychology, Faculty of Natural Sciences, University of Stirling, Stirling, UK.

Email: magda.mustile@stir.ac.uk

\begin{abstract}
The ability to safely negotiate the world on foot takes humans years to develop, reflecting the extensive cognitive demands associated with real-time planning and control of walking. Despite the importance of walking, methodological limitations mean that surprisingly little is known about the neural and cognitive processes that support ambulatory motor control. Here, we report mobile EEG data recorded from 32 healthy young adults during real-world ambulatory obstacle avoidance. Participants walked along a path while stepping over expected and unexpected obstacles projected on the floor, allowing us to capture the dynamic oscillatory response to changes in environmental demands. Compared to obstacle-free walking, time-frequency analysis of the EEG data revealed clear neural markers of proactive and reactive forms of movement control (occurring before and after crossing an obstacle), visible as increases in frontal theta and centroparietal beta power respectively. Critically, the temporal profile of changes in frontal theta allowed us to arbitrate between early selection and late adaptation mechanisms of proactive control. Our data show that motor plans are updated as soon as an upcoming obstacle appears, rather than when the obstacle is reached. In addition, regardless of whether motor plans required updating, a clear beta rebound was present after obstacles were crossed, reflecting the resetting of the motor system. Overall, mobile EEG recorded during real-world walking provides novel insight into the cognitive and neural basis of dynamic motor control in humans, suggesting new routes to the monitoring and rehabilitation of motor disorders such as dyspraxia and Parkinson's disease.
\end{abstract}

\section{KEYWOR D S}

action planning, embodied cognition, gait adaptation, motor control, walking 


\section{INTRODUCTION}

Moving safely through the environment while walking requires continual monitoring and adjustment of planned behaviour, including the ability to make fast online motor transformations in response to dynamic changes such as the appearance of unexpected obstacles. The skill of negotiating the constraints of the environment while walking is inherently complex; it develops slowly throughout infancy (Mowbray \& Cowie, 2020) and is progressively lost in aging and motor impairments such as Parkinson's disease (Holtzer et al., 2014; Peterson \& Horak, 2016). The gradual reduction in cognitive resources and motor control that occurs with aging and disease means that it becomes increasingly difficult to respond effectively to obstacles that are encountered while walking. Indeed, falls associated with stumbling or tripping over objects represent a critical factor in the increased mortality rates that are seen for elderly and neurological patients (Kovacs, 2005; Tinetti et al., 1988; Weerdesteyn et al., 2006). Given the complexity and fragility of the processes involved in walking, it is clearly important to identify the neural processes supporting cognitive control during walking and obstacle avoidance, generating new targets for clinical practice (Alexander \& Hausdorff, 2008; Peterson et al., 2016).

Over the last decade, growing research interest in human ambulation has led to the extensive recording of EEG (the electroencephalogram) during active walking on treadmills (Petersen et al., 2012; Severens et al., 2012; Gwin et al., 2010; Gwin et al., 2011; Gramann et al., 2011; Wagner et al., 2012; Wagner et al., 2016; Wagner et al., 2019; Seeber et al., 2014; Seeber et al., 2015). Recorded from electrodes placed on the scalp, EEG provides a non-invasive representation of oscillatory brain activity produced during task performance, allowing the identification of functionally dissociable cortical mechanisms that drive human behaviour (Buzsáki \& Draguhn, 2004). To date, EEG studies of walking have revealed the activation of several 'prefrontal' brain signals that are activated during the approach to an obstacle, explained as the recruitment of additional cognitive resources. For example, Haefeli et al. (2011) recorded EEG while participants walked on a treadmill, finding increased activity over frontal areas in response to an acoustic signal that warned of upcoming obstacles. Similar findings have been reported using mobile functional near-infrared spectroscopy (fNIRS). For example, Maidan et al. (2018) reported a higher hemodynamic response in the prefrontal cortex when participants had to prepare to step over unanticipated obstacles (compared to hemodynamic responses during normal walking), independent of the size of the objects. These findings have been extended by a recent EEG investigation of walking on a treadmill (Nordin et al., 2019). Nordin et al. reported increased power in low-frequency oscillations (ranging from 3 to $13 \mathrm{~Hz}$, i.e., delta, theta and alpha) while participants walked at different speeds and stepped over foam obstacles (appearing from behind a curtain placed at the front of the treadmill). These oscillatory brain changes were widespread across the scalp, with source localisation suggesting the engagement of a distributed cortical network (i.e. supplementary motor, premotor and posterior parietal areas). Furthermore, on the basis of timing information, Nordin et al. (2019) argued that obstacle avoidance involved identifying the obstacle and interrupting the gait cycle (associated with early engagement of premotor and supplementary motor areas) and then planning the foot placements required to cross the obstacle (associated with later activation of posterior parietal cortex).

Wider interest in the processes involved in goal-directed behaviour have led to the development of theoretical models of cognitive control-and these models provide a framework for understanding ambulatory control. Notably, studies of cognitive control by Braver (Braver, 2012; see also Pezzullo \& Ognibene, 2012) have characterised two broad stages of control processing. First, when a behaviour is planned, proactive control processes are employed to respond to potential sources of interference, allowing the original goal to be reached. Importantly, in theory, proactive control processes can occur at different times, reflecting either early selection or late adaptation of planned behaviour. Second, after an unexpected event has occurred, reactive control processes are employed to allow recovery from the interference and return to the original goal. Markedly similar distinctions between proactive and reactive control mechanisms have also emerged from studies on human balance (Bhatt et al., 2018; Horak, 2006; ShumwayCook \& Woollacott, 2007). Proactive strategies are used to anticipate the loss of balance (due to some source of interference), when the body has enough space and time to predict the upcoming interference and adjust motor plans. By contrast, reactive strategies involve compensatory adjustments to restore postural control and balance after unexpected events.

Although the theoretical distinction between proactive and reactive control strategies was not developed in relation to ambulatory control per se, the distinction is nonetheless clearly relevant for understanding the processes supporting obstacle avoidance during walking. Indeed, the neural signals observed in studies of treadmill walking can be readily interpreted within this 'dual mode' framework (Dual Mechanisms of Control, Braver, 2012). For example, Nordin et al. (2019) reported modulation of low-frequency oscillations linked to supplementary motor cortex and posterior parietal cortex. Both signals occurred before unexpected obstacles, consistent with the operation of a proactive control mechanism that allows planned behaviour to be refined. Moreover, the timing of the posterior parietal signal suggests that the adjustment was made shortly before the obstacle was encountered, which is suggestive of a late adaptation form of proactive control.

To our knowledge there is no equivalent evidence of EEG markers of reactive control during obstacle avoidance. 
However, there is wide evidence for reactive control mechanisms after movement. For example, EEG studies have revealed post-movement increases in beta power $(13-30 \mathrm{~Hz})$, described as the beta rebound, a marker of reactive control (Liebrand et al., 2017). Evidence from the wider literature on movement control reveals that beta power over sensory motor regions is enhanced when the predictions of an incoming stimulus are violated (Arnal et al., 2011) and after forcibly interrupted movements (Alegre et al., 2008; Heinrichs-Graham et al., 2017), suggesting a mechanism that re-calibrates the motor system after a movement (Engel \& Fries, 2010; Kilavik et al., 2013; Pfurtscheller et al., 1996). Thus, although reactive control mechanisms have not been demonstrated during obstacle avoidance, changes in beta power may index the operation of such mechanisms.

The recent emergence of mobile EEG (Gramann et al., 2011, 2014; Ladouce et al., 2017; Makeig et al., 2009) represents a particularly important development for researchers interested in walking, not least because mobile techniques significantly extend the range of contexts in which brain signals can be studied (e.g. see Park et al., 2018; Park et al., 2015). Critically, using mobile EEG technology makes it possible to monitor the brain while participants navigate natural environments, taking walking research away from the use of treadmills and out of the laboratory (see Ladouce et al., 2019; Park \& Donaldson, 2019, for recent examples). As a result, the neuro-cognitive processes supporting walking can now be studied in the real world, offering an entirely new embodied perspective to the understanding of human behaviour and motor impairments (which had been previously limited to non-ecological settings and fairly uninformative tasks; cf. Ladouce et al., 2017; McFadyen et al., 2017). Furthermore, the high temporal resolution of EEG (i.e. millisecond accuracy), combined with wireless portability, makes mobile EEG ideally suited to capturing the rapid cortical responses that occur in response to dynamic stimuli (Makeig et al., 2009).

As far as we are aware, currently there is no direct evidence for EEG markers of proactive and reactive control processes during real-world ambulatory obstacle avoidance. Thus, our primary aim in the current study is to ask whether it is possible to identify neural signals of proactive and reactive control during real-world obstacle avoidance. To address this issue, we recorded EEG while participants walked freely along a 10-m-long path. Critically, we manipulated the presentation of obstacles across trials, providing participants with more or less time and space to prepare for a response to the obstacle. Obstacles were absent, always present at the start of the action, or appeared up ahead after a short or long delay. In addition, we manipulated the available time and space that participants had to adjust their gait when negotiating the environment, while allowing the walking task to remain as natural as possible. Based on the literature reviewed above, and as a test of the dual-mode framework
(Braver, 2012), we predicted that distinct proactive and reactive control mechanisms should be identifiable based on their temporal dynamics.

As well as demonstrating that neural markers of movement control can be identified during natural walking, we also examined two specific hypotheses. First, by varying the time and space that participants had to prepare for an obstacle we were able to arbitrate between early selection and late adaptation mechanisms of proactive control. Current evidence (cf. Nordin et al., 2019) is not sufficient to differentiate between early and late proactive control mechanisms before an obstacle is overcome. Here, we predict that proactive control processes will operate as soon as information about an upcoming obstacle becomes available (i.e. early selection). Put simply, the high temporal resolution of EEG data should allow us to reveal the precise temporal dynamics of proactive control during walking. Second, by varying the opportunity to anticipate and prepare before adjusting to an obstacle, we aimed to test whether reactive control processes during walking are indexed by changes in beta power (the so-called beta rebound). That is, we tested whether reactive control processes are specifically associated with recovery after a change in a motor plan, in order to reset the previous state. As we show below, mobile EEG does indeed capture and characterise the dynamic engagement of proactive and reactive control processes during real-world ambulatory obstacle avoidance.

\section{2 | MATERIALS AND METHODS}

This study was approved by the local ethics committee and conformed to standards set by the Declaration of Helsinki. Thirty-two healthy participants (21 females and 11 males; age range $=19-65$; mean age $=32.1$ years, $S D=11.6$ years) took part in the experiment. All participants were right handed (self-reported) and gave their written informed consent before the experiment.

The experimental design involved four conditions (as depicted in Figure 1) in which participants walked along a 10-m-long carpet, passing through a series of infrared laser beams that recorded their location and controlled the presentation of obstacles (visible as a coloured patch projected onto the floor that had to be stepped over). In the "no adjustment" condition, no obstacle was presented, and participants simply walked across the room. In the "preset adjustment" condition, obstacles were present at the start of each trial, placed at a fixed location $250 \mathrm{~cm}$ from the first laser beam. In the "immediate adjustment" condition, walking through the laser beam would trigger the presentation of an obstacle, displayed $160 \mathrm{~cm}$ in front of the participant. Finally, in the "delayed adjustment" condition, walking through the laser beam once again triggered the presentation of an obstacle, presented $310 \mathrm{~cm}$ in front of the participant. The participants were always instructed to 


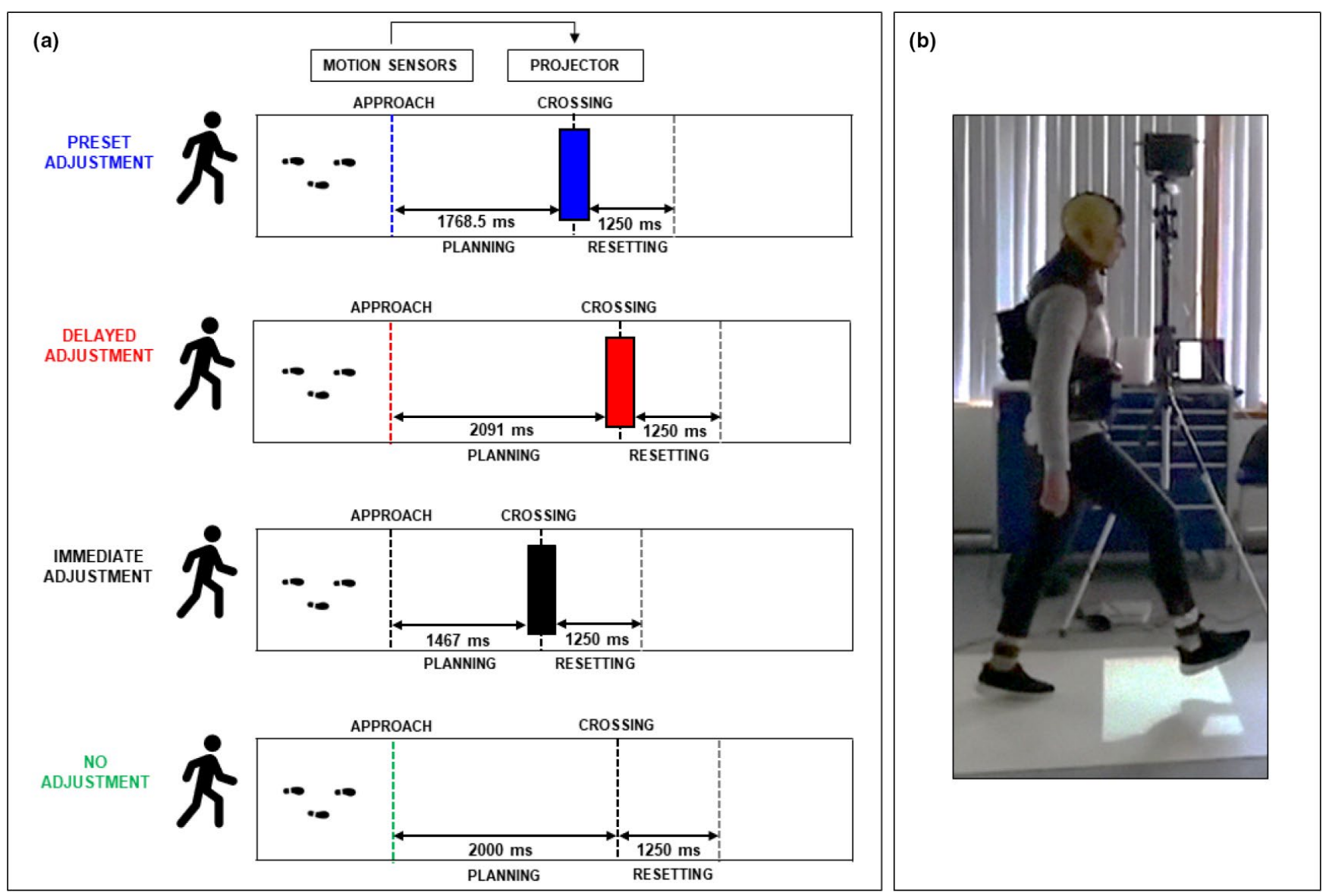

F I G URE 1 Panel a) Representation of the experimental conditions, indicated with different colors (from top to bottom, respectively: blue, preset adjustment; red, delayed adjustment; black, immediate adjustment; green, no adjustment). For each condition, the median duration (in ms) of the planning phase (between participants) is reported inside each path between the approach and the crossing dashed lines. Panel b) Photograph of a participant, wearing the mobile EEG equipment, as they cross an obstacle

walk straight across the room, to maintain a comfortable pace and to step over any obstacle presented in front of them. Each crossing of the room corresponded to an individual trial, and on reaching the end of the carpet participants were asked to turn around and walk back across the room in the same way. The video projector and laser beams were arranged to allow data collection in both directions. Participants completed a total of 240 trials divided into six experimental blocks. Each block lasted around $5 \mathrm{~min}$. All conditions were presented with equal probability. Participants were given 5-10 min breaks between each experimental block, and were encouraged to request additional breaks during each block should they need this. Any systematic influence of fatigue on the data was further minimised through randomisation of condition order across participants. The overall experimental session lasted approximately $90 \mathrm{~min}$, including preparation, recording and breaks between experimental blocks.

The obstacle was presented as a white stripe $(40 \times 80 \mathrm{~cm})$ projected on a $10-\mathrm{m}$-long carpet. The obstacle presentation was controlled with a system interfacing two fixed motion sensors, placed at $230 \mathrm{~cm}$ from both ends of the carpet (directing infrared laser beams across the room, through which participants would pass). Stimulus presentation was controlled using E-prime 3.0 software (Psychology Software Tools) and a projector. The motion sensors were designed to send an input signal to the stimulus presentation software running on a laptop, using the Auxiliary I/O port of a Chronos response device (Psychology Software Tools). The laptop was connected to a projector placed at the side of the carpet. The presence and location of the obstacle presented varied on a trial-by-trial basis, depending on the experimental condition.

During each trial, the experimenter manually marked two main events (as illustrated in Figure 1): the moment that the participant crossed the beam ('Approach') and the moment when the participant was over the obstacle ('Crossing'). These two points provided temporal markers for use within the analysis of the EEG data that identified a planning phase (before the obstacle was encountered) and a resetting phase (after the obstacle was encountered).

Participants also wore foot sensor insoles (Pedar-x System, novel.de), a bluetooth pressure distribution measuring system for monitoring local loads between the foot and the shoe. The data of gait parameters were not recorded in all participants of this study and are not reported here. 


\subsection{EEG acquisition and analysis}

EEG data were recorded from $32 \mathrm{Ag} / \mathrm{AgCl}$ electrodes connected to a portable amplifier (ANT-neuro, Enschede, The Netherlands). Electrodes were positioned according to the International 10-20 system (FP1, FPz, FP2, F7, F3, Fz, F4, F8, FC5, FC1, FC2, FC6, M1, T7, C3, Cz, C4, T8, M2, CP5, CP1, CP2, CP6, P7, P3, Pz, P4, P8, POz, O1, Oz, O2) with $\mathrm{AFz}$ electrode as ground and $\mathrm{CPz}$ electrode as reference. The electrode impedances were reduced below $5 \mathrm{k} \Omega$ before the recording. During recording EEG data were sampled at $500 \mathrm{~Hz}$ and bandpass filtered at $0.01-250 \mathrm{~Hz}$. EEG data analyses were performed using custom scripts written in MATLAB 2019a (The MathWorks) incorporating EEGLAB toolbox (Delorme \& Makeig, 2004). Data from the mastoid channels (M1 and M2) were removed from the analysis, and all remaining EEG data were filtered using a 0.1 to $40 \mathrm{~Hz}$ bandpass filter. EEG channels with prominent artefacts were automatically selected (kurtosis > 5 SDs) and interpolated, and all channels were then re-referenced to the average.

An extended infomax Independent Component Analysis (ICA, Makeig et al., 1996) was performed to identify and remove non-brain signals. Artifactual ICs scalp maps were selected through SASICA (Semi-Automated Selection of Independent Components of the electroencephalogram for Artifact Correction, Chaumon et al., 2015) combined with the ADJUST (Automatic EEG artefact Detection based on the Joint Use Of Spatial and Temporal features, Mognon et al., 2011) and MARA (Multiple Artifact Rejection Algorithm, Winkler et al., 2011) plugins. These methods provide objective means of evaluating artifactual signals that, due to their multi-dimensionality and heterogeneity, can be difficult to interpret and identify in raw EEG. Consequently, we used the following measures: Autocorrelation, Focal Components, Signal-to-Noise Ratio, Dipole Fit Residual Variance, Correlation with other channel(s), ADJUST and MARA. Detailed descriptions of each of these methods are available in the wider literature, however, for clarity here we briefly describe what each procedure achieves, highlighting any parameter settings employed.

The 'Autocorrelation' measure detects noisy components with weak autocorrelation (e.g. muscle artefacts) with a threshold of 2 SDs and a lag of $20 \mathrm{~ms}$. The 'Focal Components' measure detects components that are too focal and thus unlikely to correspond to neural activity (e.g. bad channels or muscle artefacts) with a threshold of 2 SDs. The 'Signal-to-Noise Ratio' measure (0-Inf, -Inf 0, threshold ratio $=1$ ) is used to identify components with weak signalto-noise ratio that occur between the baseline and the time window of interest. The 'Dipole Fit Residual Variance' measure (threshold $=15 \%$ ) detects components with high residual variance after subtraction of the forward dipole model. The 'Correlation with other channel(s)' measure detects components whose time course correlates with any channel(s), with a threshold of 4 SDs. The ADJUST (Mognon et al., 2011) and MARA (Winkler et al., 2011) plugins automatically compute spatial and temporal features (using pre-set parameters) to classify components as artefacts. The resulting ICs scalp maps (or topographies) were further visually inspected to identify ICs with low residual variance $(<15 \%)$. Across conditions an average (mean $\pm S D$ ) of $5.85 \pm 1.97$ of non-artifactual ICs were retained for analysis.

After artefacts were removed, the remaining data were segmented into epochs relative to the step over the obstacle (i.e. the 'Crossing' event, which was defined as time 0), producing a $-3,500 \mathrm{~ms}$ to $2,000 \mathrm{~ms}$ time window. Since the latency of different trials was affected by a great deal of variability within and between participants, single-trial spectrograms were time warped to the median latency (across participants) of the 'Approach' event using linear interpolation. In order to have the same number of trials, 40 trials were randomly selected for each condition. Epochs that exceeded the bounding values within the epochs, that is, in which the latency of the 'Approach' event exceeded the limit of $-2,500 \mathrm{~ms}$ from the 'Crossing', were excluded (i.e. trials in which the participant was very slow to walk along the carpet). Epochs were further visually inspected to identify trials that still appeared to be contaminated by prominent muscular artefacts and these were manually removed. Across conditions, an average (mean $\pm S D$ ) of $37 \pm 2.07$ epochs were included in the subsequent analysis, resulting in $7.5 \%$ of trials being excluded. Event Related Spectral Perturbations (ERSPs) were obtained by computing the mean difference between single-trial log spectrograms for each channel, for each participant, relative to the mean baseline spectrum (from $-3,000 \mathrm{~ms}$ preceding to $1,500 \mathrm{~ms}$ following the obstacle stepping).

\subsection{Statistical analysis}

Midline single-channel spectrograms $(\mathrm{Fc}, \mathrm{Cz}$ and $\mathrm{POz}$; Figure 2) were visually inspected to identify prominent changes in the spectral power across conditions. Informed by our hypothesis and visual inspection of the topographic maps of theta (Figures 3 and 4) and beta (Figure 5) power, we identified frontal (FC1, Fz and FC2 channels), central (CP1, $\mathrm{Cz}$ and $\mathrm{CP} 2)$ and parietal (P3, POz and P4) locations that captured the effects of interest. Finally, in order to examine the time course of spectral changes before and after the obstacle, the planning (from $-1,750 \mathrm{~ms}$ to $-250 \mathrm{~ms}$ ) and the resetting (from $250 \mathrm{~ms}$ to $1,250 \mathrm{~ms}$ ) periods were divided into a series of successive $500 \mathrm{~ms}$ time windows. Three different repeated measures ANOVAs with three within factors (Experimental Condition, Time Window and Scalp Location) were performed to examine the power modulation across the planning and the resetting phases for each frequency band. 


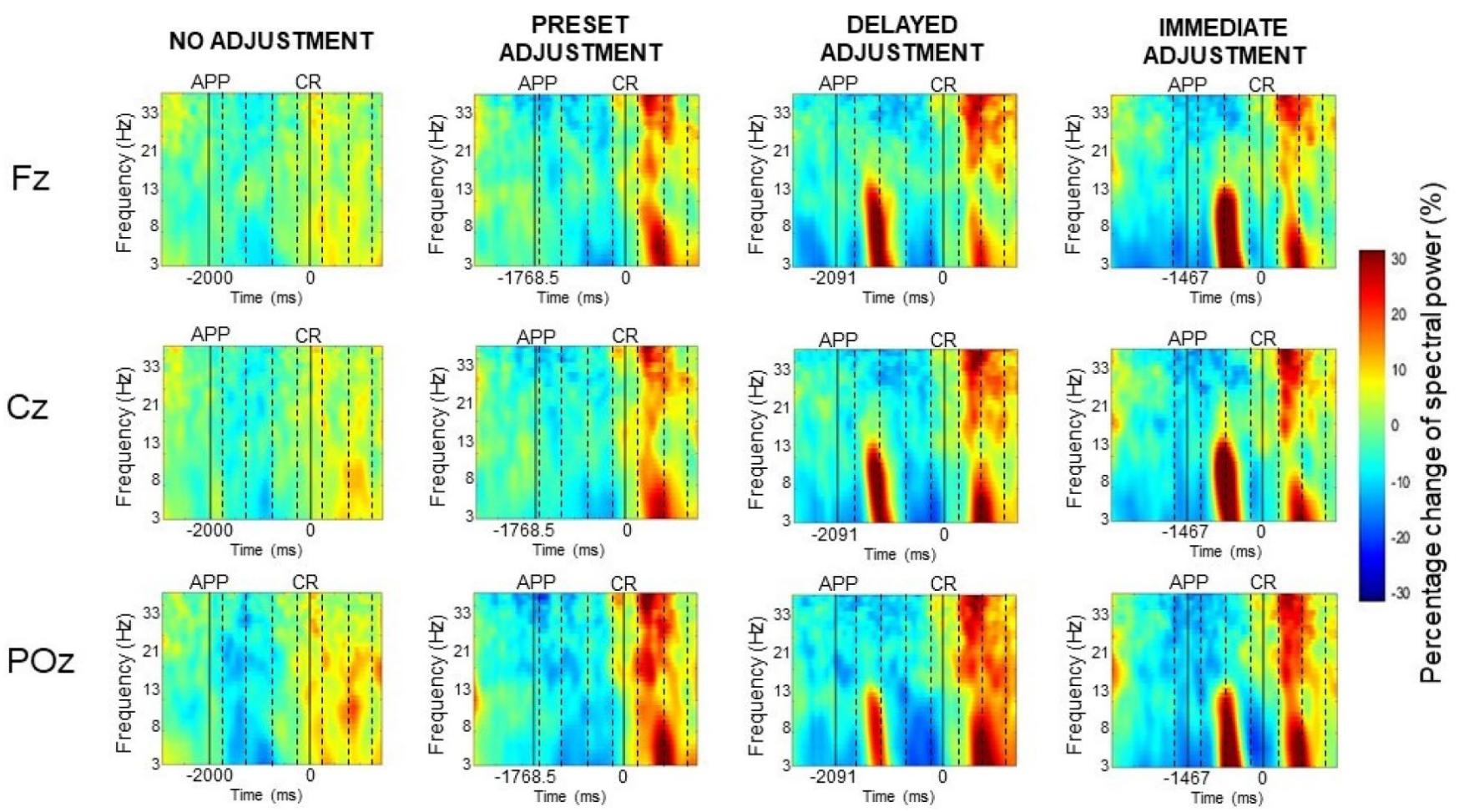

F I G U RE 2 Time warped spectrograms at electrodes Fz, Cz, and POz for each experimental condition. Vertical solid black lines represent the 'Approach' (APP) and the 'Crossing' (CR, time 0) events, respectively. Vertical dashed lines represent time windows included in the analysis. On the $x$-axis (time in $\mathrm{ms}$ ), the median latencies of the timing of the Approach point are reported for each condition. The lowest frequency shown is $3 \mathrm{~Hz}$, the highest is $35 \mathrm{~Hz}$. Colors indicate the relative change of power from the baseline (\%). Blue colors represent decrease of power; red colors indicate increase of power

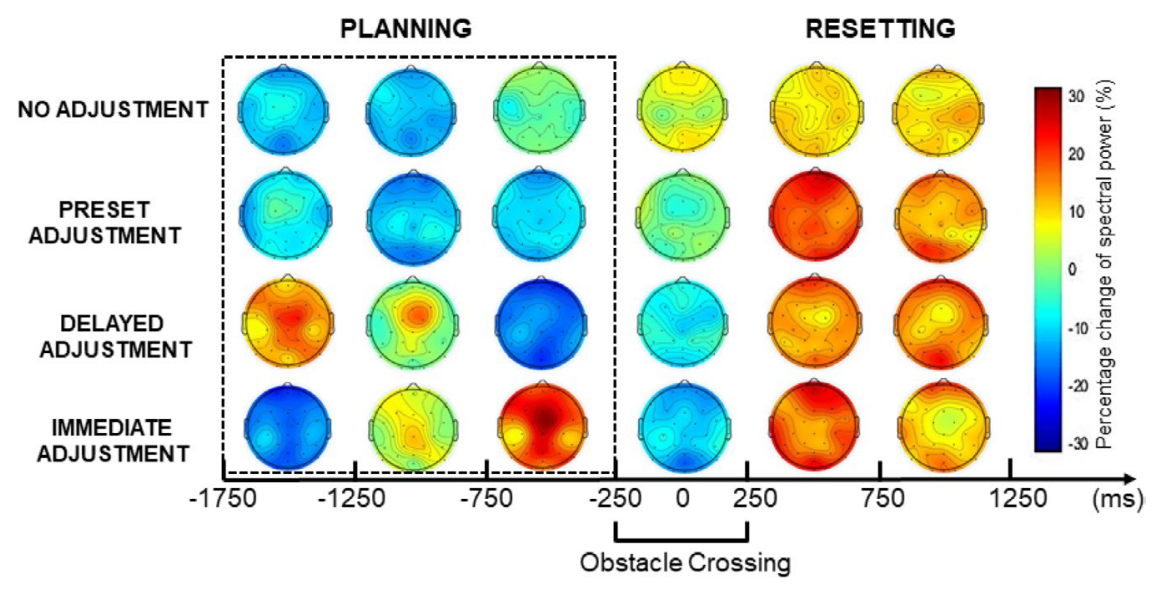

FIGURE 3 Topographic maps illustrating the temporal dynamics of theta power across conditions and time windows. The dashed rectangle around the scalp maps before time 0 , indicates the time windows included in the planning phase

Significance level was set at $p<.05$ and, where the sphericity assumption was violated, the Greenhouse-Geisser method was used to correct the degrees of freedom. Post-hoc paired samples $t$-tests were adjusted for multiple comparisons using Bonferroni correction.

\section{3 | RESULTS}

Midline time warped spectrograms (Figure 2) revealed a transient change in the spectral power of theta $(4-7 \mathrm{~Hz})$ and beta $(13-35 \mathrm{~Hz})^{1}$ frequency bands, occurring after the 'Approach' and before 'Crossing' and differently distributed across conditions. Below the results for each frequency band are presented separately for the planning and the resetting phases.

\footnotetext{
${ }^{1}$ Analysis of alpha $(8-12 \mathrm{~Hz})$ oscillations is not included in this manuscript in order to focus analysis on the more prominent theta and beta bands. However, for interest, alpha oscillations mirror the patterns of theta oscillations that we report here.
} 
FIGURE 4 The time course of percentage changes from the baseline in theta power across the experimental conditions (group mean, with standard errors indicated by shading) shown for a representative electrode (FC1). Dashed lines represent the median latency of the 'Approach' event, that matches the same color of the conditions indicated by the key. Solid vertical black line indicates the 'Crossing' event (time 0). The black rectangle indicates the time windows included in the analysis of the planning phase

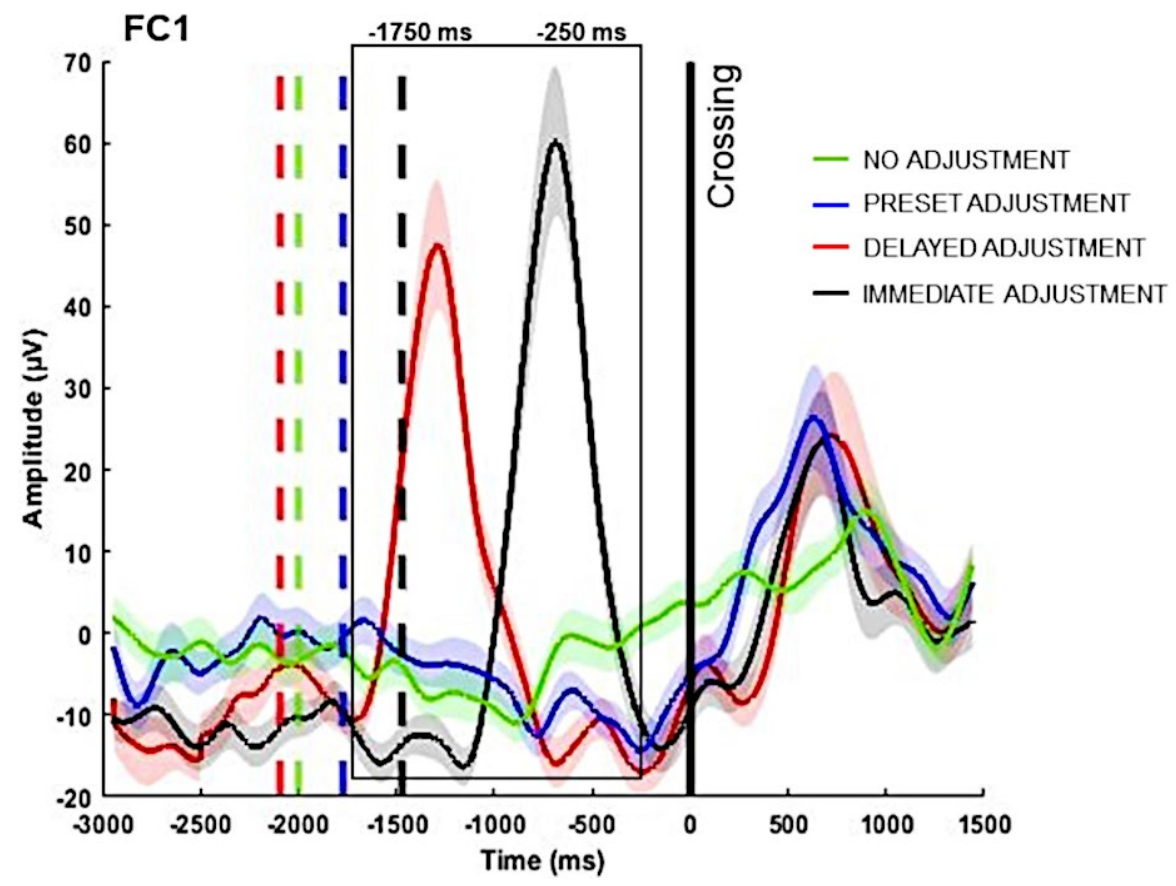

F I G U RE 5 Topographic maps

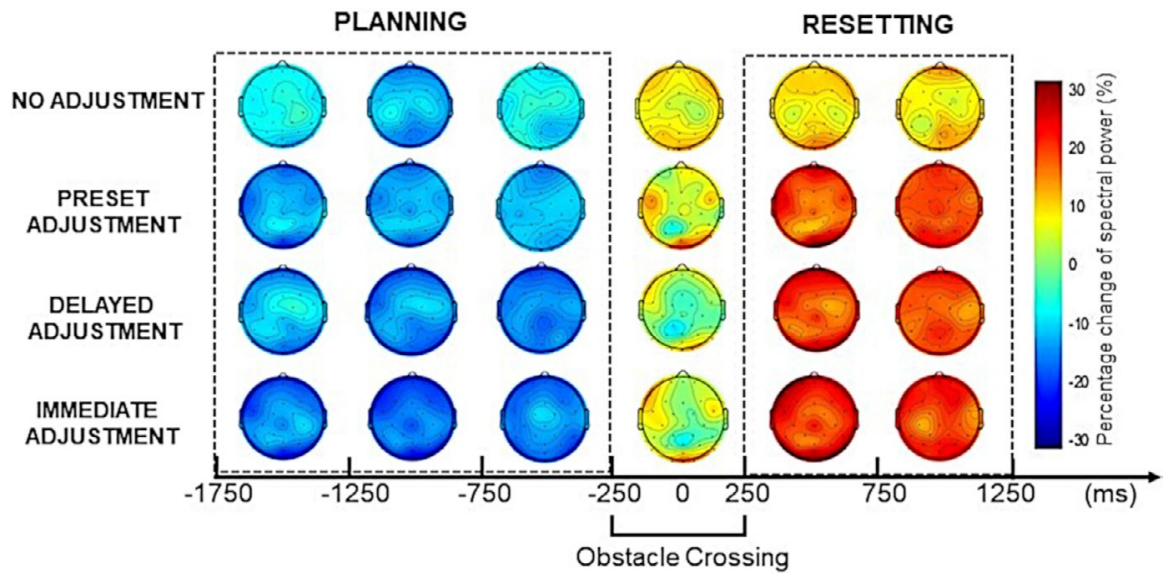

\section{1 | Planning}

\subsection{1 | Theta}

The ANOVA indicated that changes in the theta spectral power were significantly different across experimental conditions $\left[F_{(1,31)}=14.645, p<.001, \eta_{\mathrm{p}}^{2}=0.321\right]$. Post-hoc paired sample $t$-tests revealed that the theta increase was significantly stronger both in the immediate adjustment [immediate vs. no adjustment: $t_{(31)}=6.150, p<.001 ;$ immediate vs. pre-set: $t_{(31)}=5.374, p<.001$; immediate vs. delayed: $\left.t_{(31)}=2.142, p<.05\right]$ and in the delayed adjustment condition [delayed vs. no adjustment: $t_{(31)}=-4.235, p<.001$; delayed vs. pre-set: $t_{(31)}=-2.811, p<.01$ ], but similar in the pre-set adjustment and no adjustment conditions $(p=.375)$. A main effect of Scalp Location $\left[F_{(1,31)}=8.302, p<.001\right.$, $\left.\eta_{\mathrm{p}}^{2}=0.211\right]$ revealed that the theta increase was more pro- nounced at frontal compared to parietal $\left[t_{(31)}=3.733\right.$, $p<.001]$ and central $\left[t_{(31)}=-2.154, p<.05\right]$ electrodes, and decreased strongly in parietal compared to central $\left[t_{(31)}=2.138, p<.05\right]$ electrodes.

A significant interaction between Experimental Condition and Time Window $\left[F_{(1,31)}=37.313, p<.001, \eta_{\mathrm{p}}^{2}=0.546\right.$; Figures 2 and 3] confirmed that the timing of the increase in theta power was consistent with the appearance of the obstacle in the immediate and delayed adjustment conditions. As shown in Figure 3, a significantly stronger theta increase occurred firstly in the delayed adjustment after the obstacle appeared [ $-1,750 \mathrm{~ms}$ to $-1,250 \mathrm{~ms}$; delayed vs. no adjustment: $t_{(31)}=-6.007, p<.001$; delayed vs. pre-set: $t_{(31)}=-4.150, p<.001 ;$ delayed vs. immediate: $\left.t_{(31)}=-5.598, p<.001\right]$ and decreased more in the immediate compared to pre-set adjustment condition $\left[t_{(31)}=-3.248\right.$, $p<.01]$. In the following time window $(-1,250 \mathrm{~ms}$ to 
$-750 \mathrm{~ms}$ ), the theta increase became stronger in the immediate adjustment condition [immediate vs. no adjustment: $t_{(31)}=4.922, p<.001$; immediate vs. pre-set: $t_{(31)}=4.432$, $p<.001]$ but was still present in the delayed adjustment condition [delayed vs. no adjustment: $t_{(31)}=-6.052, p<.001$; delayed vs. pre-set: $\left.t_{(31)}=-3.345, p<.01\right]$. In the last time window the theta increase was stronger in the immediate adjustment condition [immediate vs. no adjustment: $t_{(31)}=5.902, p<.001$; immediate vs. pre-set: $t_{(31)}=6.904$, $p<.001$; immediate vs. delayed: $\left.t_{(31)}=10.882, p<.001\right]$, but the decrease was stronger in the delayed adjustment condition [delayed vs. no adjustment: $t_{(31)}=7.586, p<.001$; delayed vs. pre-set: $\left.t_{(31)}=2.163, p<.05\right]$ and in the pre-set adjustment conditions [pre-set vs. no adjustment: $\left.t_{(31)}=3.602, p<.001\right]$. Post-hoc $t$-tests revealed no statistical differences between pre-set adjustment and no adjustment conditions during the first two time windows $(p>.05)$ of the planning phase. No other main effect or interaction reached statistical significance $(p>.05)$.

\subsection{2 | Beta}

Although the ANOVA did not show a main effect of Experimental Condition, the decrease in beta power was stronger in the immediate adjustment condition (mean $=-9.69 \pm 7.09 \mu \mathrm{V}$ ), followed by the delayed adjustment condition $($ mean $=-9.08 \pm 6.73 \mu \mathrm{V})$, the pre-set adjustment condition (mean $=-8.34 \pm 6.72 \mu \mathrm{V}$ ) and no adjustment condition (mean $=-5.43 \pm 7.28 \mu \mathrm{V}$ ). A main effect of Scalp Location $\left[F_{(1,31)}=4.183, p<.05, \eta_{\mathrm{p}}^{2}=0.119\right]$ revealed that a stronger decrease in beta power occurred in central (mean $=-8.43 \pm 4.66 \mu \mathrm{V}$ ) and parietal (mean $=-8.80 \pm 5.03 \mu \mathrm{V}$ ) electrodes compared to frontal (mean $=-7.19 \pm 4.82 \mu \mathrm{V}$ ) electrodes, although post-hoc paired sample $t$-tests showed only one statistically significant difference [parietal vs. frontal: $t_{(31)}=2.589, p<.05$ ]. A significant interaction between Time Windows and Experimental Condition $\left[F_{(1,31)}=2.919, p<.05, \eta_{\mathrm{p}}^{2}=0.086\right.$; Figure 6] showed that beta decrease was signifi- cantly stronger in all obstacle conditions compared to no adjustment in the last time window [ -750 to $-250 \mathrm{~ms}$; no adjustment vs. immediate: $t_{(31)}=-2.876, p<.01$; no adjustment vs. delayed: $t_{(31)}=4.997, p<.001$; no adjustment vs. pre-set: $t_{(31)}=3.742$, $p<.001]$. A significant interaction between Scalp Location and Time Window $\left[F_{(1,31)}=4,595, p<.01, \eta_{\mathrm{p}}^{2}=0.129\right]$ revealed that beta decrease was initially stronger in parietal electrodes [time $-1,750$ to $-1,250 \mathrm{~ms}$; parietal vs. frontal: $\left.t_{(31)}=2.219, p<.05\right]$ but later $(-750$ to $-250 \mathrm{~ms})$ when the participants were approaching the obstacle became stronger in central electrodes compared to frontal $\left[t_{(31)}=-3.395\right.$, $p<.01]$ and parietal $\left[t_{(31)}=-3.475, p<.01\right]$ electrodes. No other main effect or interaction reached statistical significance $(p>.05)$.

\section{2 | Resetting phase}

\subsection{1 | Beta}

The ANOVA revealed a main effect of Experimental Condition $\left[F_{(1,31)}=9.912, p<.001, \eta_{\mathrm{p}}^{2}=0.242\right]$ on beta modulation during the resetting phase. The increase in beta power was stronger in the all obstacle conditions compared to no adjustment condition [no adjustment vs. immediate: $t_{(31)}=4.525, p<.001 ;$ no adjustment vs. delayed: $t_{(31)}=-5.113, p<.001$; no adjustment vs. pre-set: $\left.t_{(31)}=-4.062, p<.001\right]$. Additionally, the beta increase was stronger in the delayed adjustment condition compared to the immediate adjustment condition $\left[t_{(31)}=-2.461, p<.05\right]$ but not compared to pre-set adjustment condition [immediate vs. pre-set: $p=.839$; delayed vs. pre-set: $p=.258$ ]. A main

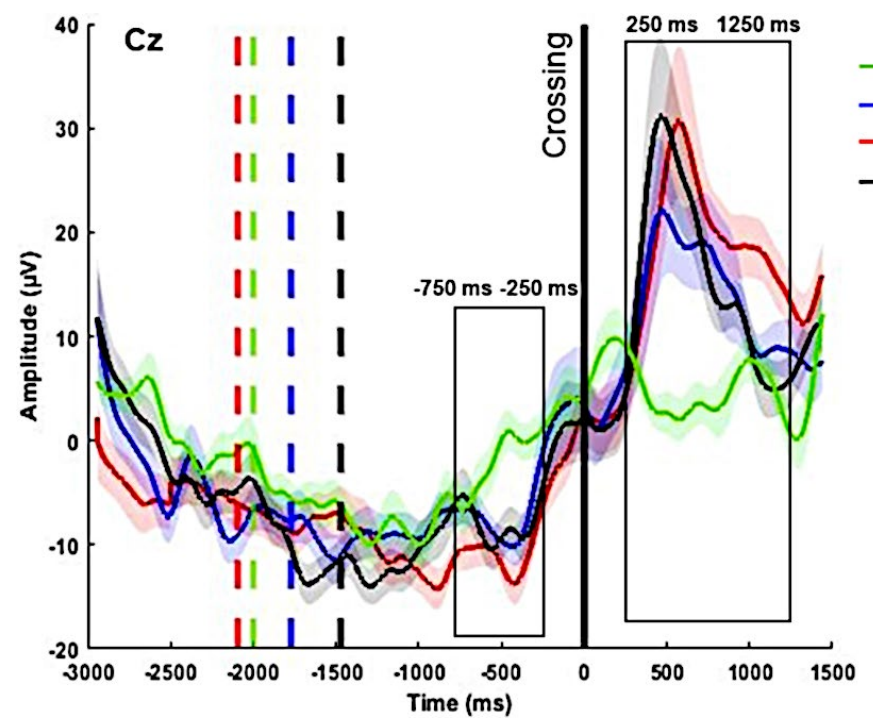

F I G URE 6 The time course of percentage changes from the baseline in beta power across the experimental conditions (group mean, with standard errors indicated by shading) shown for a representative electrode $(\mathrm{Cz})$. Solid vertical black line indicates the 'Crossing' event (time 0). The black rectangles indicate the time windows in which we found significant differences between conditions $(-750 \mathrm{~ms}$ to $-250 \mathrm{~ms}$ and $250 \mathrm{~ms}$ to $1,250 \mathrm{~ms}$ respectively) 
effect of Scalp Location $\left[F_{(1,31)}=4.028, p<.05, \eta_{\mathrm{p}}^{2}=0.115\right]$ revealed that the beta increase was stronger in parietal compared to central $\left[t_{(31)}=-2.143, p<.05\right]$ and frontal $\left[t_{(31)}=-2.143, p<.01\right]$ electrodes. No other main effect or interaction reached statistical significance $(p>.05)$.

\section{4 | DISCUSSION}

To our knowledge, this is the first mobile EEG investigation of real-world ambulatory obstacle avoidance. Our primary aim was to assess whether neural signals associated with proactive and reactive forms of cognitive control could be detected during naturalistic movements, using the temporal dynamics of the neural response to dissociate the cognitive processes involved. In short, the results revealed distinct neural markers of proactive and reactive control, distinguishable in frequency (i.e. in theta or beta, respectively) and in time (i.e. present before or after the obstacle, respectively). This neural dissociation is, to our knowledge, the first evidence in support of the dual-mode account of cognitive control (cf. Braver, 2012) during real-world walking. Furthermore, the temporal dynamics of the neural response observed while participants approached an obstacle revealed that, within the cognitive process of proactive control, selection is made early. These data are therefore unique in identifying a specific time point at which movement preparation processes occur, a finding that has implications for continuous models of proactive motor control (e.g. Pezzullo \& Ognibene, 2012). Below we discuss the implications of these findings for the understanding of each of these control processes, as well as considering technical and practical implications, limitations and recommendations for future studies.

We start by focusing on proactive control mechanisms. As predicted, the analysis revealed a clear neural marker of proactive control: transient increases in theta power over frontal scalp locations during the planning phase, consistent with the timing of the unexpected obstacles' appearance on the path. Analysis of the spectral EEG data revealed that the increase in theta power was largest when participants had less time and space available to change their gait before stepping over an obstacle (i.e. in the immediate adjustment condition). By contrast, this modulation was substantially absent when participants walked without encountering any obstacle (i.e. in the no adjustment condition) or when they could see the obstacle in advance (i.e. in the pre-set adjustment condition). Taken together, therefore, the pattern across conditions strongly suggests that increases in frontal theta observed during walking mark a proactive cognitive control mechanism that is engaged in response to unexpected obstacles.

More importantly, the temporal dynamics of theta showed that the increase in power was linked to the appearance of the obstacle, suggesting an early selection mechanism is at play (Braver, 2012) within proactive control (Pezzullo \& Ognibene, 2012). Nordin et al. (2019) investigated the brain dynamics during obstacle avoidance, and concluded that changes in posterior parietal alpha, theta and delta power (i.e. 3-13 Hz) occurred just two steps before the obstacle, providing support for a late adaptation model of control. Analogous oscillatory changes were found by Nordin and colleagues in premotor and supplementary motor areas that preceded posterior parietal cortex activation. However, the limited length of the treadmill belt in the study by Nordin et al. (2019) prevents safe conclusions from being drawn regarding the timing of these changes in relation to crossing the obstacle. By contrast, the present study clearly demonstrates that increases in frontal theta are not related to the time that an obstacle is overcome, but instead to the time that the walker becomes aware of an obstacle. Thus, the present results provide clear evidence in favour of an early selection mechanism underlying proactive control.

According to dual-mode theory, proactive control operates through mechanisms which actively maintain relevant information until the behaviour is accomplished (Braver, 2012). In theory, therefore, the continuous maintenance of goalrelevant information supporting complex behaviour in the real world must require the recruitment of substantial cognitive resources. In situations where a planned action needs to be maintained rather than performed immediately, proactive control must operate in a flexible and cost-efficient manner, updating relevant information to ensure that the appropriate action can take place at the right time (Pezzullo \& Ognibene, 2012). As noted above, however, in the context of negotiating unexpected obstacles, our mobile EEG findings pinpoint the specific timing of frontal theta in relation to the appearance of the to-be-avoided obstacle-revealing an early selection mechanism within proactive control. Support for our account of proactive control can be found in the task switching literature (Cooper et al., 2015, 2017, 2019), where cognitive control has been divided into two stages: an early component, which ensures the preparation and the updating of relevant information facing the change, and a later component that reflects response readiness (Cooper et al., 2015, 2017, 2019). Cooper and colleagues found that, in the context of task switching, frontal theta reflects an early cognitive control mechanism. Taken together, therefore, evidence suggests that increases in frontal theta power index an 'early' proactive mechanism, associated with preparing for an upcoming change, regardless of when the action is to be executed.

Having discussed changes in theta power and the timing of proactive control that occurs before an obstacle is encountered, we now turn to changes in beta power that occur as the obstacle is reached. During the planning phase, a greater decrease in beta power was observed at electrodes over sensorimotor areas when the participants 
had to step over obstacles, compared to when there was no obstacle to avoid. As unobstructed walking involves a more basic negotiation of one's environment compared to obstacle avoidance, the greater decrease in beta band power likely reflects a state of increased motor readiness, which is needed in order to negotiate the obstacle without interrupting the walking cycle. Furthermore, the magnitude of the decrease in beta power was larger over parietal scalp locations during the earlier stages of movement, but larger at central electrodes when the participants approached the obstacle.

Decreases in parietal beta power have previously been observed during visually guided step adjustments (Wagner et al., 2012) and motor preparation of finger movements (Mars et al., 2007). Central beta power decreases have previously been observed in humans during active walking (Presacco et al., 2011; Seeber et al., 2014; Wagner et al., 2012; Wieser et al., 2010) and cycling (Jain et al., 2013; Storzer et al., 2016). It is well established that changes in beta power over sensorimotor brain regions index motor activation, thought to reflect the planning and the execution of voluntary movements (Neuper et al., 2006; Pfurtscheller \& Berghold, 1989; Pfurtscheller \& Lopes da Silva, 1999). Consequently, the temporal evolution of beta power in the present study points towards the operation of a sequential mechanism, which initially recruits sensorimotor integration and spatial representation processes and, at a later stage, movement planning processes. Moreover, neither the temporal evolution nor magnitude of the decrease in beta power were notably different when gait adjustments were present or were triggered by the presentation of unexpected obstacles. This aspect of the data likely reflects the relatively low difficulty of stepping over obstacles in the present study. In addition, however, it also suggests that the updating of motor plans, presumably reflected in theta increases, is not necessarily reflected in greater primary sensorimotor activity.

We now turn to consideration of processes that occur once an obstacle has been negotiated. During the resetting phase we observed robust transient power changes in beta (the so-called post-movement beta rebound; cf. Jurkiewicz et al., 2006; Pfurtscheller et al., 2005; Pfurtscheller \& SolisEscalante, 2009). The beta rebound is typically observed over sensorimotor areas after motor execution or motor imagery (Pfurtscheller et al., 2005; Pfurtscheller \& SolisEscalante, 2009) and it is believed to reflect an active recalibration process that takes place after a change in the state of the motor system (Engel \& Fries, 2010; Kilavik et al., 2013; Pfurtscheller et al., 1996). Notably, studies of cognitive control (e.g. during task switching and go/no-go paradigms; see Cooper et al., 2019 and Liebrand et al., 2017, respectively) suggest the beta rebound seen over prefrontal and sensorimotor areas is an index of reactive control (cf. Liebrand et al., 2017). Accordingly, the presence of the beta rebound in the current study suggests that reactive control processes are also engaged during naturalistic walking, to restore the motor system to its previous state. Critically, and consistent with our hypothesis, increases in beta power were present only when gait adjustments were required (in order to step over the obstacle), but were absent when there was no obstacle to avoid.

The current study design also allowed us to explore whether the beta rebound was modulated by the amount of time participants had to adjust their gait. As such, the beta rebound may be modulated by the motor demands placed when adapting gait. Although the beta rebound was clearly present after negotiating the obstacle, this index of recovery was not more pronounced when obstacles appeared while walking compared to when the obstacle was present at the start of the journey. Modulation was also evident when obstacles appeared while walking: the beta rebound was stronger when the participants had more time to adjust their gait before stepping over the obstacle. Visual inspection of the shape of the beta rebound waveform suggests, however, that this effect may be due to the fact that the beta rebound was prolonged when participants had more time. A recent study has suggested that the duration of the beta rebound is increased after temporally protracted movements (Fry et al., 2016). Accordingly, the modulation of beta rebound appears to signify the longer engagement of the motor system when an unexpected obstacle appeared at a great distance from the participants.

\section{5 | PRACTICAL IMPLICATIONS, TECHNICAL CONSIDERATIONS AND FUTURE DIRECTIONS}

The overarching objective of the present study was to demonstrate the relevance and utility of using mobile EEG in real-world investigations, in particular for detecting neural correlates of natural behaviour that cannot be captured in traditional laboratory settings (Gramann et al., 2011, 2014; Ladouce et al., 2017; Makeig et al., 2009). Despite extensive development of new hardware solutions (i.e. dry electrodes, Lopez-Gordo et al., 2014, or dual-layer EEG caps, Nordin et al., 2019) and tools for signal processing (i.e. independent component analysis, Makeig et al., 1996), to date mobile technologies have not typically been used to test cognitive theories (Ladouce et al., 2017). Here, by revealing neurocognitive indices of proactive and reactive control, we show that mobile EEG can be used to characterise the complex pattern of processes that are engaged when humans encounter obstacles while walking.

The technical challenges of reducing motion artefacts during natural movements, and the need to integrate the information from multiple devices, differentiate the mobile EEG approach from traditional laboratory-based EEG. A further 
obvious difference lies in the number of channels employed: here, we used 32 mobile EEG channels, rather than the 64 or 128 channels commonly used in laboratory-based systems. Inevitably, the methodological constraints associated with mobile EEG have implications for data processing, analysis and interpretation. For example, the use of fewer electrodes results in the extraction of fewer independent components during the pre-processing stage than in equivalent laboratorybased studies. Equally, the use of a restricted electrode montage also precludes using EEG to identify the neural sources of the recorded signal, preventing any strong conclusions about the specific brain regions responsible. More importantly, mobile EEG data tend to be "noisier" in terms of movement and muscle artefact than traditional laboratorybased EEG data, making it more difficult to identify brain signals. To address this issue, we relied heavily on automatic artefact rejection procedures to provide an objective evaluation of brain and non-brain signals. Despite the limitations associated with mobile data collection, the present experiment clearly demonstrates that cognitive processing can be assessed during real-world behaviour.

Another challenge for mobile EEG research lies in the presentation and manipulation of real-world stimuli within the context of a structured experimental design. For example, in the present study, "obstacles" were images projected onto the floor, which allowed us to easily manipulate the position and predictability of the objects while maintaining a naturalistic and safe environment for participants. Although our paradigm was not entirely natural, lights projected onto the floor have previously been used in behavioural studies of obstacle avoidance (Chen et al., 1996; Salazar-Varas et al., 2015). In addition, the use of $2 \mathrm{D}$ (rather than 3D) obstacles could potentially interfere with natural leg mechanics while walking. Critically, however, our aim was to identify the cognitive processes relating to overcoming expected and unexpected obstacles, rather than to explore gait patterns per se. It is nevertheless of interest for future studies to examine whether the introduction of real 3D obstacles significantly influences the engagement of control processes. Similarly, future studies will need to examine the impact of variability in the temporal-spatial features of walking (i.e. changes in speed, acceleration or direction) and the participants' performance (i.e. successful vs. unsuccessful obstacle avoidance) in order to identify any links between brain cortical dynamics and gait patterns.

To fully investigate all of the factors that influence walking would, of course, require a more complex paradigm than was employed here, for example, via the introduction of routes that allow walkers to turn repeatedly. Other developments will require the use of additional sensors, for example, to allow EEG recordings to be time-stamped based on the pattern and timing of heel strikes that are made during walking. Even without additional technical and methodological development, the present findings highlight the exciting opportunities that now exist for studying neurodegenerative and developmental disorders (such as Parkinson's disease and dyspraxia, respectively), where understanding real-world behaviours is critical. Negotiating obstacles in the real-world requires us to allocate attention, detect relevant constraints and flexibly adapt motor behaviours, which is challenging for elderly or Parkinson's disease patients who often experience gait impairments that increase the risk of falls and mortality (Kovacs, 2005; Tinetti et al., 1988; Weerdesteyn et al., 2006). Studies that aimed to identify neural markers of Parkinson's disease and gait dynamics are presently limited, being restricted to simple tasks (i.e. finger tapping, Stegemöller et al., 2016, 2017) or indirect methods such as kinematics recording (Galna et al., 2010; Vitório et al., 2010). By contrast, mobile EEG can provide direct insight into the neural and cognitive processes that are affected by disorders, addressing the actual real-world situations that are problematic for patients.

For rehabilitation of motor disorders following brain injury or as a consequence of neurodegenerative diseases such as Parkinson's disease, it is particularly important to have an understanding of the cognitive processes involved in the complex, dynamic, modality integrated reality of realworld behaviour. As such, identifiable neural markers of real-world behaviour offer novel pathways towards tailored neuro-rehabilitation approaches for motor disorders in particular. Such evidence-based cognitive rehabilitation strategies could, for example, use neurofeedback or non-invasive brain stimulation and the online acquisition of cognitive neural markers in offering tailored and ecological diagnostics and rehabilitation processes for patients affected by various neurological aetiologies.

\section{6 | CONCLUSION}

Our study demonstrates that mobile EEG can be used to capture the dynamic oscillatory responses associated with the neuro-cognitive processes of that are engaged while negotiating real-world environments. We demonstrated that naturalistic obstacle avoidance is mediated by proactive and reactive cognitive control processes, reflected in the dynamics of theta and beta oscillations. In particular, the temporal brain dynamics of frontal theta revealed that proactive control during unexpected obstacle avoidance is associated with an early selection mechanism. Furthermore, we showed that motor readiness is mediated by beta power decreases which were evident when pre-set or externally triggered gait adjustments were needed in order to step over an obstacle. With regards to reactive control, we identified a robust beta rebound after obstacles were crossed, demonstrating that real-world negotiation of the environment requires finely tuned resetting of the motor system. Taken 
together, these mobile EEG data provide a new way to examine the neuro-cognitive processes supporting walking in particular, and of applying an embodied mobile cognition perspective to the understanding of human behaviour in general. The findings are also relevant towards a better understanding of motor impairments in more naturalistic contexts and should inform the development of novel neuro-rehabilitation approaches.

\section{7 | DATA AVAIBILITY STATEMENT}

The data supporting the findings of this study are available at https://osf.io/9gnad/?view_only=0052ce57c18749a9bc60 4ba8380712e6 and at http://hdl.handle.net/11667/169.

\section{ACKNOWLEDGEMENTS}

We would like to thank technicians Chris Grigson, Stephen Stewart and Catriona Bruce for their help with the experimental setup and recordings. This work is supported by a scolarship from the University of Stirling and a reseach grant from SINAPSE (Scottish Imaging Network: A Platform for Scientific excellence). GL is supported by the Wellcome Trust [209209/Z/17/Z].

\section{CONFLICT OF INTERESTS}

The authors declare no conflict of interest or relationship, financial or otherwise.

\section{AUTHOR CONTRIBUTIONS}

Author contributions: M.M., S.L., D.K., G.L., M.G.E, D.I.D. and M.I. conception and design of the study; M.M. performed the experiment; M.M. and S.L. analysed data; M.M., S.L., D.K., M.G.E., D.I.D. and M.I. interpreted results and all authors contributed to the manuscript.

\section{PEER REVIEW}

The peer review history for this article is available at https:// publons.com/publon/10.1111/ejn.15120.

\section{ORCID}

Magda Mustile (iD https://orcid.org/0000-0002-7903-3184

Dimitrios Kourtis iD https://orcid.org/0000-0003-2535-6196

Simon Ladouce (iD https://orcid.org/0000-0001-6760-6240

Gemma Learmonth iD https://orcid.org/0000-0003-4061-4464

Martin G. Edwards iD https://orcid.org/0000-0003-4532-2436

David I. Donaldson (iD https://orcid.org/0000-0002-8036-3455

Magdalena Ietswaart iD https://orcid.org/0000-0003-4576-9393

\section{REFERENCES}

Alegre, M., Alvarez-Gerriko, I., Valencia, M., Iriarte, J., \& Artieda, J. (2008). Oscillatory changes related to the forced termination of a movement. Clinical Neurophysiology, 119, 290-300. https://doi. org/10.1016/j.clinph.2007.10.017

Alexander, N. B., \& Hausdorff, J. M. (2008). Guest editorial: Linking thinking, walking, and falling. The Journals of Gerontology Series A, Biological Science, 63, 1325-1328.

Arnal, L. H., Wyart, V., \& Giraud, A. L. (2011). Transitions in neural oscillations reflect prediction errors generated in audiovisual speech. Nature Neuroscience, 14, 797.

Bhatt, T., Patel, P., Dusane, S., DelDonno, S. R., \& Langenecker, S. A. (2018). Neural mechanisms involved in mental imagery of slipperturbation while walking: A preliminary fMRI study. Frontiers in Behavioural Neurosciences, 12, 203.

Braver, T. S. (2012). The variable nature of cognitive control: A dual mechanisms framework. Trends in Cognitive Sciences, 16, 106-113.

Buzsáki, G., \& Draguhn, A. (2004). Neuronal Oscillations in Cortical Networks. Science, 304, 1926-1929. https://doi.org/10.1126/scien ce. 1099745

Chaumon, M., Bishop, D. V., \& Busch, N. A. (2015). A practical guide to the selection of independent components of the electroencephalogram for artifact correction. Journal of Neuroscience Methods, 250, 47-63.

Chen, H. C., Schultz, A. B., Ashton-Miller, J. A., Giordani, B., Alexander, N. B., \& Guire, K. E. (1996). Stepping over obstacles: Dividing attention impairs performance of old more than young adults. The Journals of Gerontology Series A: Biological Sciences and Medical Sciences, 51, M116-M122. https://doi.org/10.1093/ gerona/51A.3.M116

Cooper, P. S., Karayanidis, F., McKewen, M., McLellan-Hall, S., Wong, A. S., Skippen, P., \& Cavanagh, J. F. (2019). Frontal theta predicts specific cognitive control-induced behavioural changes beyond general reaction time slowing. NeuroImage, 189, 130-140. https://doi. org/10.1016/j.neuroimage.2019.01.022

Cooper, P. S., Wong, A. S., Fulham, W. R., Thienel, R., Mansfield, E., Michie, P. T., \& Karayanidis, F. (2015). Theta frontoparietal connectivity associated with proactive and reactive cognitive control processes. NeuroImage, 108, 354-363. https://doi.org/10.1016/j.neuro image.2014.12.028

Cooper, P. S., Wong, A. S., McKewen, M., Michie, P. T., \& Karayanidis, F. (2017). Frontoparietal theta oscillations during proactive control are associated with goal-updating and reduced behavioral variability. Biological Psychology, 129, 253-264.

Delorme, A., \& Makeig, S. (2004). EEGLAB: An open source toolbox for analysis of single-trial EEG dynamics including independent component analysis. Journal of Neuroscience Methods, 134, 9-21.

Engel, A. K., \& Fries, P. (2010). Beta-band oscillations—-signalling the status quo? Current Opinion in Neurobiology, 20, 156-165.

Fry, A., Mullinger, K. J., O'Neill, G. C., Barratt, E. L., Morris, P. G., Bauer, M., Folland, J. P., \& Brookes, M. J. (2016). Modulation of post-movement beta rebound by contraction force and rate of force development. Human Brain Mapping, 37, 2493-2511.

Galna, B., Murphy, A. T., \& Morris, M. E. (2010). Obstacle crossing in people with Parkinson's disease: Foot clearance and spatiotemporal deficits. Human Movement Science, 29, 843-852.

Gramann, K., Ferris, D. P., Gwin, J., \& Makeig, S. (2014). Imaging natural cognition in action. International Journal of Psychophysiology, 91, 22-29.

Gramann, K., Gwin, J. T., Ferris, D. P., Oie, K., Jung, T., Lin, C., Liao, L., \& Makeig, S. (2011). Cognition in action: Imaging brain/body dynamics in mobile humans. Reviews in the Neurosciences, 22, 593-608. 
Gwin, J. T., Gramann, K., Makeig, S., \& Ferris, D. P. (2010). Removal of movement artifact from high-density EEG recorded during walking and running. Journal of Neurophysiology, 103, 3526-3534.

Gwin, J. T., Gramann, K., Makeig, S., \& Ferris, D. P. (2011). Electrocortical activity is coupled to gait cycle phase during treadmill walking. NeuroImage, 54, 1289-1296. https://doi.org/10.1016/j. neuroimage.2010.08.066

Haefeli, J., Vögeli, S., Michel, J., \& Dietz, V. (2011). Preparation and performance of obstacle steps: Interaction between brain and spinal neuronal activity. European Journal of Neuroscience, 33, 338-348.

Heinrichs-Graham, E., Kurz, M. J., Gehringer, J. E., \& Wilson, T. W. (2017). The functional role of post-movement beta oscillations in motor termination. Brain Structure and Function, 222, 3075-3086.

Holtzer, R., Epstein, N., Mahoney, J. R., Izzetoglu, M., \& Blumen, H. M. (2014). Neuroimaging of mobility in aging: A targeted review. The Journals of Gerontology. Series A, Biological Sciences, 69, $1375-1388$.

Horak, F. B. (2006). Postural orientation and equilibrium: What do we need to know about neural control of balance to prevent falls? Age and Ageing, 35, ii7-ii11. https://doi.org/10.1093/ageing/af1077

Jain, S., Gourab, K., Schindler-Ivens, S., \& Schmit, B. D. (2013). EEG during pedaling: Evidence for cortical control of locomotor tasks. Clinical Neurophysiology, 124, 379-390. https://doi.org/10.1016/j. clinph.2012.08.021

Jurkiewicz, M. T., Gaetz, W. C., Bostan, A. C., \& Cheyne, D. (2006). Post-movement beta rebound is generated in motor cortex: Evidence from neuromagnetic recordings. NeuroImage, 32, 1281-1289. https://doi.org/10.1016/j.neuroimage.2006.06.005

Kilavik, B. E., Zaepffel, M., Brovelli, A., MacKay, W. A., \& Riehle, A. (2013). The ups and downs of beta oscillations in sensorimotor cortex. Experimental Neurology, 245, 15-26.

Kovacs, C. R. (2005). Age-related changes in gait and obstacle avoidance capabilities in older adults: A review. Journal of Applied Gerontology, 24, 21-34. https://doi.org/10.1177/0733464804 271279

Ladouce, S., Donaldson, D. I., Dudchenko, P. A., \& Ietswaart, M. (2017). Understanding minds in real-world environments: Toward a mobile cognition approach. Frontiers in Human Neuroscience, 10, 694.

Ladouce, S., Donaldson, D. I., Dudchenko, P. A., \& Ietswaart, M. (2019). Mobile EEG identifies the re-allocation of attention during real-world activity. Scientific Reports, 9, 1-10.

Liebrand, M., Pein, I., Tzvi, E., \& Krämer, U. M. (2017). Temporal dynamics of proactive and reactive motor inhibition. Frontiers in Human Neuroscience, 11, 204.

Lopez-Gordo, M. A., Sanchez-Morillo, D., \& Valle, F. P. (2014). Dry EEG electrodes. Sensors, 14, 12847-12870.

Maidan, I., Shustak, S., Sharon, T., Bernad-Elazari, H., Geffen, N., Giladi, N., Hausdorff, J. M., \& Mirelman, A. (2018). Prefrontal cortex activation during obstacle negotiation: What's the effect size and timing? Brain Cognition, 122, 45-51.

Makeig, S., Bell, A. J., Jung, T. P., \& Sejnowski, T. J. (1996). Independent component analysis of electroencephalographic data. Advances in Neural Information Processing Systems, 6, 145-151.

Makeig, S., Gramann, K., Jung, T. P., Sejnowski, T. J., \& Poizner, H. (2009). Linking brain, mind and behavior. International Journal of Psychophysiology, 73, 95-100.
Mars, R. B., Piekema, C., Coles, M. G., Hulstijn, W., \& Toni, I. (2007). On the programming and reprogramming of actions. Cerebral Cortex, 17, 2972-2979.

McFadyen, B. J., Gagné, M. È., Cossette, I., \& Ouellet, M. C. (2017). Using dual task walking as an aid to assess executive dysfunction ecologically in neurological populations: A narrative review. Neuropsychol. Rehabil., 27, 722-743.

Mognon, A., Jovicich, J., Bruzzone, L., \& Buiatti, M. (2011). ADJUST: An automatic EEG artifact detector based on the joint use of spatial and temporal features. Psychophysiology, 48, 229-240.

Mowbray, R., \& Cowie, D. (2020). Mind your step: Learning to walk in complex environments. Experimental Brain Research, 238, $1455-1465$.

Neuper, C., Wörtz, M., \& Pfurtscheller, G. (2006). ERD/ERS patterns reflecting sensorimotor activation and deactivation. Progress in Brain Research, 159, 211-222.

Nordin, A. D., Hairston, W. D., \& Ferris, D. P. (2019). Human electrocortical dynamics while stepping over obstacles. Scientific Reports, $9,1-12$.

Park, J. L., \& Donaldson, D. I. (2019). Detecting the neural correlates of episodic memory with mobile EEG: Recollecting objects in the real world. NeuroImage, 193, 1-9. https://doi.org/10.1016/j.neuro image.2019.03.013

Park, J. L., Dudchenko, P. A., \& Donaldson, D. I. (2018). Navigation in real-world environments: New opportunities afforded by advances in mobile brain imaging. Frontiers in Human Neuroscience, 12, 361.

Park, J. L., Fairweather, M. M., \& Donaldson, D. I. (2015). Making the case for mobile cognition: EEG and sports performance. Neuroscience and Biobehavioral Reviews, 52, 117-130.

Petersen, T. H., Willerslev-Olsen, M., Conway, B. A., \& Nielsen, J. B. (2012). The motor cortex drives the muscles during walking in human subjects. Journal of Physiology, 590, 2443-2452.

Peterson, D. S., \& Horak, F. B. (2016). Neural control of walking in people with parkinsonism. Physiology, 31, 95-107. https://doi. org/10.1152/physiol.00034.2015

Peterson, D. S., King, L. A., Cohen, R. G., \& Horak, F. B. (2016). Cognitive contributions to freezing of gait in Parkinson disease: Implications for physical rehabilitation. Physical Therapy, 96, 659670. https://doi.org/10.2522/ptj.20140603

Pezzulo, G., \& Ognibene, D. (2012). Proactive action preparation: Seeing action preparation as a continuous and proactive process. Motor Control, 16, 386-424. https://doi.org/10.1123/mcj.16.3.386

Pfurtscheller, G., \& Berghold, A. (1989). Patterns of cortical activation during planning of voluntary movement. Electroencephalography and Clinical Neurophysiology, 72, 250-258. https://doi. org/10.1016/0013-4694(89)90250-2

Pfurtscheller, G., \& Da Silva, F. L. (1999). Event-related EEG/MEG synchronization and desynchronization: Basic principles. Clinical Neurophysiology, 110, 1842-1857. https://doi.org/10.1016/S1388 -2457(99)00141-8

Pfurtscheller, G., Neuper, C., Brunner, C., \& Da Silva, F. L. (2005). Beta rebound after different types of motor imagery in man. Neuroscience Letters, 378, 156-159.

Pfurtscheller, G., \& Solis-Escalante, T. (2009). Could the beta rebound in the EEG be suitable to realize a "brain switch"? Clinical Neurophysiology, 120, 24-29. https://doi.org/10.1016/j. clinph.2008.09.027

Pfurtscheller, G., Stancak, A. Jr, \& Neuper, C. (1996). Post-movement beta synchronization. A correlate of an idling motor area? 
Electroencephalography and Clinical Neurophysiology, 98, 281293. https://doi.org/10.1016/0013-4694(95)00258-8

Presacco, A., Goodman, R., Forrester, L., \& Contreras-Vidal, J. L. (2011). Neural decoding of treadmill walking from noninvasive electroencephalographic signals. Journal of Neurophysiology, 106, 1875-1887.

Salazar-Varas, R., Costa, Á., Iáñez, E., Úbeda, A., Hortal, E., \& Azorín, J. M. (2015). Analyzing EEG signals to detect unexpected obstacles during walking. J Neuroeng Rehabil, 12, 101. https://doi. org/10.1186/s12984-015-0095-4

Seeber, M., Scherer, R., Wagner, J., Solis-Escalante, T., \& Müller-Putz, G. R. (2014). EEG beta suppression and low gamma modulation are different elements of human upright walking. Frontiers in Human Neuroscience, 8, 485.

Seeber, M., Scherer, R., Wagner, J., Solis-Escalante, T., \& Müller-Putz, G. R. (2015). High and low gamma EEG oscillations in central sensorimotor areas are conversely modulated during the human gait cycle. NeuroImage, 112, 318-326. https://doi.org/10.1016/j.neuro image.2015.03.045

Severens, M., Nienhuis, B., Desain, P., \& Duysens, J. (2012) Feasibility of measuring event related desynchronization with electroencephalography during walking. In 2012 Annual International Conference of the IEEE Engineering in Medicine and Biology Society (pp. 2764-2767). IEEE.

Shumway-Cook, A., \& Woollacott, M. H. (2007). Motor control: Translating research into clinical practice, : . Lippincott Williams \& Wilkins.

Stegemöller, E. L., Allen, D. P., Simuni, T., \& MacKinnon, C. D. (2016). Motor cortical oscillations are abnormally suppressed during repetitive movement in patients with Parkinson's disease. Clinical Neurophysiology, 127, 664-674. https://doi.org/10.1016/j. clinph.2015.05.014

Stegemöller, E. L., Allen, D. P., Simuni, T., \& MacKinnon, C. D. (2017). Altered premotor cortical oscillations during repetitive movement in persons with Parkinson's disease. Behavioral Brain Research, 317 , 141-146.

Storzer, L., Butz, M., Hirschmann, J., Abbasi, O. et al (2016). Bicycling and walking are associated with different cortical oscillatory dynamics. Frontiers in Human Neuroscience, 10, 61.

Tinetti, M. E., Speechley, M., \& Ginter, S. F. (1988). Risk factors for falls among elderly persons living in the community. New England Journal of Medicine, 319, 1701-1707.
Vitório, R., Pieruccini-Faria, F., Stella, F., Gobbi, S., \& Gobbi, L. T. B. (2010). Effects of obstacle height on obstacle crossing in mild Parkinson's disease. Gait \& Posture, 31, 143-146. https://doi. org/10.1016/j.gaitpost.2009.09.011

Wagner, J., Makeig, S., Gola, M., Neuper, C., \& Müller-Putz, G. (2016). Distinct $\beta$ band oscillatory networks subserving motor and cognitive control during gait adaptation. Journal of Neuroscience, 36, 2212-2226.

Wagner, J., Martínez-Cancino, R., \& Makeig, S. (2019). Trial-by-trial source-resolved EEG responses to gait task challenges predict subsequent step adaptation. NeuroImage, 199, 691-703. https://doi. org/10.1016/j.neuroimage.2019.06.018

Wagner, J., Solis-Escalante, T., Grieshofer, P., Neuper, C., Müller-Putz, G., \& Scherer, R. (2012). Level of participation in robotic-assisted treadmill walking modulates midline sensorimotor EEG rhythms in able-bodied subjects. NeuroImage, 63, 1203-1211. https://doi. org/10.1016/j.neuroimage.2012.08.019

Weerdesteyn, V., Rijken, H., Geurts, A. C., Smits-Engelsman, B. C., Mulder, T., \& Duysens, J. (2006). A five-week exercise program can reduce falls and improve obstacle avoidance in the elderly. Gerontology, 52, 131-141. https://doi.org/10.1159/000091822

Wieser, M., Haefeli, J., Bütler, L., Jäncke, L., Riener, R., \& Koeneke, S. (2010). Temporal and spatial patterns of cortical activation during assisted lower limb movement. Experimental Brain Research, 203, 181-191.

Winkler, I., Haufe, S., \& Tangermann, M. (2011). Automatic classification of artifactual ICA-components for artifact removal in EEG signals. Behavioral and Brain Functions, 7, 30. https://doi. org/10.1186/1744-9081-7-30

How to cite this article: Mustile M, Kourtis D, Ladouce S, et al. Mobile EEG reveals functionally dissociable dynamic processes supporting real-world ambulatory obstacle avoidance: Evidence for early proactive control. Eur J Neurosci. 2021;00:1-14. https://doi.org/10.1111/ejn.15120 